\title{
A NEW FORMALISM FOR THE COMPUTATION OF RKKY INTERACTION IN APERIODIC SYSTEMS
}

\author{
Stephan Roche $\dagger$ And Didier Mayou * \\ † Department of Applied Physics, University of Tokyo, 7-3-1 Hongo, Bunkyo-ku, Tokyo 113, Japan. \\ * LEPES-CNRS, avenue des Martyrs- BP166 38042 Grenoble France
}

\begin{abstract}
A numerical method to investigate RKKY interaction between localized spins in aperiodic materials is presented. Based on an expansion of the spectral measure on orthogonal polynomials, this approach leads to an effective framework to analyse linear response formula for the RKKY coupling in complex systems. Particularly useful in the tight-binding scheme it is used in this paper to probe the role of the local environment and the quasiperiodic potential on the interaction between magnetic spins. Interesting features are revealed and discussed within the context of anomalous localization and transport.
\end{abstract}

PACS numbers: 72.90.+y 61.44.Br 72.10.-d

\section{INTRODUCTION}

The purpose of this work is to present a new formalism based on real space recursion scheme [1] which enables to compute the so-called Rudermann Kittel Kasuya Yosida interaction (RKKY) [2 [0] an effective coupling between two localized magnetic moments, mediated by conduction electrons.

This long range oscillatory interaction is now well understood in pure metals and in weakly disordered systems, or even close to the Anderson transition. RKKY interaction has been the subjected of a great attention during the past years for instance for understanding the spin glass transition [6], magnetic long range order in high$T_{c}$ cuprates [7], or more recently because of its anomalous behavior related to giant magnetoresistance effects in magnetic multilayers [8].

As conduction electrons carry this interaction, one can wonder what happens for systems in which electronic propagation is anomalous [9] or in cases of inhomogeneous disorder ? (e.g. local inhomogeneities, correlated disorder,...). These issues may be addressed in relation to unusual RKKY interaction in quasicrystals, where the amplitude of the coupling was found to be anomalously strong and with similar values for several manganesebased quasicrystals 11 with different densities of states. Such results were found to apparently contradict the classical theory stating that RKKY should be proportional to the total DOS at Fermi level.

In the following, after reminding some general features of RKKY coupling, the method will be detailed followed by a first part addressing the numerical convergence of the algorithm. A second part is devoted to the study of the impact of local disorder or quasiperiodic potential on RKKY. This will exemplify the interest of the method.

\section{CALCULATION OF THE NON-LOCAL SUSCEPTIBILITY}

Indirect RKKY interaction stems from the coupling between localized magnetic moments and propagating electrons. If an electron in a state of energy $E<E_{F}$ undergoes a transition to a state of energy $E^{\prime}>E_{F}$ because of the coupling with the localized moment in $\left|r_{i}\right\rangle$, then a hole-electron pair is created and propagate coherently during a certain time $\tau$, with $\left|E^{\prime}-E\right| \leq \hbar / \tau$, until the pair is destroyed by diffusion on another magnetic impurity located in $\left|r_{j}\right\rangle$. Consequently the longer is the propagation time the smallest will be the vicinity around Fermi energy that account for RKKY. The generic form of the effective coupling between two magnetic impurities mediated by itinerant electrons reads :

$$
\mathcal{I}_{R K K Y}\left(r_{i}, r_{j}, E\right)=J^{2} \chi\left(r_{i}, r_{j}, E\right) \mathbf{S}_{r_{i}} \cdot \mathbf{S}_{r_{j}}
$$

with $\mathrm{J}$ is the interaction between the localized moment $\mathbf{S}_{r_{i}}$ and the spin of the itinerant electrons, and $\chi\left(r_{i}, r_{j}, E_{F}\right)$ contains the sum of all the electron-hole propagation paths from $\left|r_{i}\right\rangle$ to $\left|r_{j}\right\rangle$. RKKY is then proportional to the electronic susceptibility $\chi\left(r_{i j}\right)$ of itinerant electrons. When $J>0$ (resp. $J<0$ ), the configurations of parallel spins (resp. antiparallel) will minimize the energy promoting ferromagnetic state (resp. antiferromagnetic state). The susceptibility as a contribution of all the scattering pathes of the hole-electron pair can be written down as

$$
\chi\left(r_{i}, r_{j}\right)=-\frac{1}{2 \pi} \Im m \int_{-\infty}^{+\infty} d E G_{+}\left(r_{i}, r_{j}, E\right) G_{-}\left(r_{j}, r_{i}, E\right)
$$

introducing the retarded $\left(G_{-}\right)$and advanced Green's functions $\left(G_{+}\right)$which define the amplitude of propagation of the hole-electron pair. Note that there exists an exact sum rule between the susceptibility and the local density of states $(\mathrm{LDoS}) \sum_{j} \chi\left(r_{i}, r_{j}\right)=\rho_{i}(E)=$ 
$\frac{-2}{\pi} \Im m\left\langle r_{i}|G(z)| r_{i}\right\rangle$ which could be used as a numerical test.

In metallic systems with space dimension $\mathrm{D}$, the interaction is given by

$$
\mathcal{I}_{R K K Y}\left(r, E_{F}\right) \sim A(\mathbf{r}) \cos \left(2 k_{F} r+\delta(\mathbf{r})\right) / r^{D}
$$

which manifests a long range oscillating behavior. [2] For a free electron gas, the $A, \delta$ are independent of $\mathbf{r}$, whereas for weak disorder limit, $A(\mathbf{r})$ becomes a random but smooth function of $\mathbf{r}$ and $\delta(\mathbf{r})$ is the phase shift associated to the scattering of electrons on impurities. $\delta(\mathbf{r})$ becomes random for $r>l_{p m}$ (mean free path). It has been shown however that the mean free path is not a good measure of the typical length scale of this interaction. Indeed, the disorder average of even moments $\left\langle\chi^{2 p}\left(\left|r_{i}-r_{j}\right|\right)\right\rangle$ will contribute roughly as $\left(1 / r^{D}\right)^{1 / 2 p}$, whereas odd moments of the susceptibility will vanish exponentially over the mean free path length as expressed by the following averaged results [5]

$$
\begin{aligned}
\left\langle\chi^{2 p}\left(\left|r_{i}-r_{j}\right|\right)\right\rangle & \simeq \Omega_{p}\left(\frac{\rho^{2}\left(E_{F}\right)}{\left|r_{i}-r_{j}\right|^{2 d}}\right)^{p} \sim\left(\left\langle\chi^{2}\left(\left|r_{i}-r_{j}\right|\right)\right\rangle\right)^{p} \\
\left\langle\chi^{2 p+1}\left(\left|r_{i}-r_{j}\right|\right)\right\rangle & \simeq \exp \left(-\frac{\left|r_{i}-r_{j}\right|}{l_{p m}}\right)
\end{aligned}
$$

taking $\rho\left(E_{F}\right)$ the DoS at Fermi level, $l_{m}$ the mean free path, and $\Omega_{p}$ a constant independent of the parameter of the hamiltonian. Consequently, the average over disorder of electronic susceptibility is not sufficient for describing the correct range, amplitude and phase of the interaction, which fluctuates very much from one random configuration to another. 近,5

Let us now consider a simple argument for the RKKY coupling. If one considers a cube of length $L$ (with periodic boundary conditions) which is larger than the typical distance magnetic impurities, then the expansion of the susceptibility in a basis of eigenstates $\{|n\rangle\}$, reads :

$$
\chi\left(r_{i}, r_{j}\right)=2 \Re e \sum_{n, m} \frac{\left\langle r_{i} \mid n\right\rangle\left\langle n \mid r_{j}\right\rangle\left\langle r_{j} \mid m\right\rangle\left\langle m \mid r_{i}\right\rangle}{E_{n}-E_{m}}
$$

with $E_{n}>E_{F}>E_{m}$ and $\left\langle n \mid r_{j}\right\rangle \sim 1 / \sqrt{L^{D}}$ given that states are normalized. Given that the average spacing between energy levels for a given length is roughly $\Delta E=\left(\rho\left(E_{F}\right) L^{D}\right)^{-1}$, with $\rho(E)$ the total density of states, one can assume that the energies of the electron and hole are respectively $E_{n}=E_{F}+n \Delta E$ and $E_{m}=E_{F}-m \Delta E$ $(n, m>0)$. Consequently, the susceptibility can be expressed as

$$
\begin{aligned}
\chi\left(r_{i}, r_{j}\right) & =2\left(L^{-D}\right)^{2} \Re e \sum_{n, m} \frac{\Phi(n) \Phi^{*}(m)}{(n+m) \Delta E} \\
& =\frac{2 \rho\left(E_{F}\right)}{L^{D}} \Re e \sum_{n, m} \frac{\Phi(n) \Phi^{*}(m)}{(n+m)}
\end{aligned}
$$

where the functions $\Phi(n) \sim L^{D}\langle r \mid n\rangle\left\langle n \mid r^{\prime}\right\rangle \sim 1$. From this, one can extract the generic behavior in power laws $L^{-D}$, which only depends on the space dimension in which the system is embedded. The other part accounts for phase interferences and for instance in the case of almost free electrons (weak disorder limit), $\Phi(n)=\exp \left(i \mathbf{k} .\left(r_{i}-r_{j}\right)\right)$ so that $\Re e \Phi(n) \Phi^{*}(m) \sim$ $e^{-i k_{F}\left(r_{i}-r_{j}\right)} e^{+i k_{F}\left(r_{i}-r_{j}\right)} \sim \cos \left(2 k_{F}\left|r_{i}-r_{j}\right|\right)$ given that only states $(m, n)$ very close to the Fermi surface will contribute to the electronic response. Possible limitations of this treatment due to strong correlations between electronic levels will be discussed later on (see part V.C).

From our argument, two different effects influencing the physical properties may be drawn. On the one hand, close to metal-insulator transition peculiar electronic localization (like multifractality of eigenstates) may lead to correction of the law $1 /\left|r_{i}-r_{j}\right|^{D}$. Indeed, in quasiperiodic systems, quasicrystals or disordered fractal structures, the average displacement in time of an electron is driven by anomalous diffusion. One can wonders how electronic susceptibility is affected by such localization effects.

Besides, if the LDoS is inhomogeneous, i.e if it presents strong local fluctuations, an effect of local environment may be deduced from the equation (2) and lead to specific features for RKKY coupling. Actually, since the LDoS $|r\rangle$ can be expanded in the eigenstates basis of $\mathcal{H}$ as $\sum_{n} \delta\left(E-\varepsilon_{n}\right)|\langle r \mid n\rangle|^{2}$, a substantial increasing of the LDoS around the magnetic sites, enhancing the corresponding amplitudes $\langle r \mid n\rangle$, should also qualitatively lead to an increase of the non-local susceptibility. This has been discussed in relation with peculiar properties of AlPdMn quasicrystalline phases where the RKKY interaction was found to be anomalously large and identical for several phases with different TDoS 11 13.

\section{REAL-SPACE APPROACH OF RKKY INTERACTION}

To perform real space calculations of the non-local susceptibility, considering the Green operator $G(z)=(z-$ $\mathcal{H})^{-1}=\int_{-\infty}^{+\infty} \frac{\delta(E-\mathcal{H})}{z-E} d E$, one starts from the general form of $\chi\left(r_{i}, r_{j}\right)$ :

$2 \Re e \int_{\substack{E>E_{F} \\ E^{\prime}<E_{F}}} d E d E^{\prime} \frac{\left\langle r_{i}|\delta(E-\mathcal{H})| r_{j}\right\rangle\left\langle r_{j}\left|\delta\left(E^{\prime}-\mathcal{H}\right)\right| r_{i}\right\rangle}{E-E^{\prime}}$

The aim of the method is to determine the coefficients $\left\langle r_{i}|\delta(E-\mathcal{H})| r_{j}\right\rangle$ without exact diagonalization, usually limited to simple models and small finite 
size systems. The keypoint of the method is to use a basis of orthogonal polynomials $\left\{\mathcal{P}_{n}(E)\right\}_{n \in N}$ associated to a normalized function $\rho(E)$, referred as a model density of states. If the spectral subset of $\rho(E)$ contains the one of the real hamiltonian, it can be shown that 12 ]

$$
\delta(E-\mathcal{H})=\rho(E) \sum_{n} \mathcal{P}_{n}(E) \mathcal{P}_{n}(\mathcal{H})
$$

and the $\left\{\mathcal{P}_{n}(E)\right\}_{n \in N}$ satisfy to the orthogonality condition

$$
\int_{-\infty}^{+\infty} \rho(E) \mathcal{P}_{n}(E) \mathcal{P}_{m}(E) d E=\delta_{n m}
$$

These relations enable to write $\left\langle r_{i}|\delta(E-\mathcal{H})| r_{j}\right\rangle=$ $\rho(E) \sum_{n \in N} \mathcal{P}_{n}(E) \alpha_{i j}^{n}$ with $\alpha_{i j}^{n}=\left\langle r_{i}\left|\mathcal{P}_{n}(\mathcal{H})\right| r_{j}\right\rangle$. From these expressions, the susceptibility can be written down

$$
\begin{aligned}
\chi\left(r_{i}, r_{j}\right) & =\Re e \sum_{n m} \mathcal{I}_{m n} \alpha_{i j}^{n} \alpha_{j i}^{m} \\
\mathcal{I}_{m n} & =\int_{\substack{E>E_{F} \\
E^{\prime}<E_{F}}} \rho(E) \rho\left(E^{\prime}\right) \frac{\mathcal{P}_{m}(E) \mathcal{P}_{n}\left(E^{\prime}\right)}{E-E^{\prime}} d E d E^{\prime}
\end{aligned}
$$

Accordingly, the calculation of $\chi\left(r_{i}, r_{j}\right)$ is divided into two independent parts. Depending on the choice of polynomials, the $\mathcal{I}_{m n}$ will display particular analytical form. The other part of the susceptibility will involve a recursive evaluation of coefficients $\alpha_{i j}^{n}$ by means of the three term relations defining the orthogonal polynomials, as detailed below.

\section{A. Recursion evaluation of $\alpha_{i j}^{n}$}

One has to choose a convenient basis of orthogonal polynomials to evaluate step by step the coefficients $\alpha_{i j}^{n}$. Given that any basis of such polynomials is uniquely define by a three-term recurrence relation, generically reading $E P_{n}(E)=a_{n} \mathcal{P}_{n}(E)+b_{n} \mathcal{P}_{n+1}(E)+b_{n-1} \mathcal{P}_{n-1}(E)$ with $b_{-1}=0, n \geq 0$, and $a_{n}, b_{n}$ the associated recursion coefficients [14], the main vectors to be evaluated recursively will follow from

$$
\left|\varphi_{i}^{n}\right\rangle=\mathcal{P}_{n}(\mathcal{H})\left|r_{i}\right\rangle=\sum_{j} \alpha_{j i}^{n}\left|r_{i}\right\rangle
$$

Pratically, we will consider the Chebyshev polynomials of second order that have been already used in others contexts. Such polynomials are defined by

$$
\mathcal{P}_{n}(\mathcal{H})=\frac{1}{b}(\mathcal{H}-a) \mathcal{P}_{n}(\mathcal{H})+\mathcal{P}_{n-1}(\mathcal{H})
$$

with $\mathcal{P}_{-1}(\mathcal{H})=0$ and $\mathcal{P}_{0}(\mathcal{H})=\mathbb{1}$ and the corresponding weight is given by

$$
\rho_{a b}(E)=\frac{1}{2 \pi b^{2}} \sqrt{4 b^{2}-(E-a)^{2}}
$$

which is $\neq 0$ only for $E \in[a-2 b, a+2 b]$. The coefficients a and $\mathrm{b}$ are given by the calculated limits $a_{n \rightarrow \infty}, b_{n \rightarrow \infty}$ for the real densities of states. From the abovementionned relations, the $\left|\varphi_{i}^{n}\right\rangle$ will be given by $\left|\varphi_{i}^{n}\right\rangle=\frac{1}{b}(\mathcal{H}-a) \mid$ $\left.\varphi_{i}^{n}\right\rangle+\left|\varphi_{i}^{n-1}\right\rangle$ and $\left|\varphi_{i}^{-1}\right\rangle=0,\left|\varphi_{i}^{0}\right\rangle=\left|r_{i}\right\rangle$. In the tight-binding scheme $\mathcal{H}=\sum_{p q} \gamma_{p q}\left|r_{p}\right\rangle\left\langle r_{q}\right|$, one shows that the $\alpha_{i j}^{n}$ coefficients have to be evaluated recursively through $\left(\alpha_{p j}^{-1}=0, \alpha_{p j}^{0}=\delta_{p j}, \forall p\right)$

$$
\alpha_{i j}^{n+1}=\frac{1}{b}\left(\sum_{p} \alpha_{i p}^{n} \gamma_{p j}-a \alpha_{i j}^{n}\right)-\alpha_{i j}^{n-1}
$$

\section{B. Calculation of $\mathcal{I}_{m n}$ for the Chebyshev polynomials}

Let us now proceed to the calculation of $\mathcal{I}_{m n}$ for the Chebyshev polynomials of second order. First we rearrange the general form

$$
\mathcal{I}_{m n}=\int_{\substack{E>E_{F} \\ E^{\prime}<E_{F}}} \rho(E) \rho\left(E^{\prime}\right) \frac{\mathcal{P}_{m}(E) \mathcal{P}_{n}\left(E^{\prime}\right)}{E-E^{\prime}} d E d E^{\prime}
$$

by noticing that the factor $1 /\left(E-E^{\prime}\right)$ can be written as

$$
\begin{aligned}
\oint_{\Gamma} \frac{d z}{(z-E)\left(z-E^{\prime}\right)} & =\frac{2 i \pi}{E-E^{\prime}}\left(\Theta\left(E^{\prime}-E_{F}\right) \Theta\left(E_{F}-E\right)\right. \\
& \left.-\Theta\left(E-E_{F}\right) \Theta\left(E_{F}-E^{\prime}\right)\right)
\end{aligned}
$$

with the Heaviside function $(\Theta(x)=0, x<0$ and $\Theta(x)=$ $1, x>0)$ and the contour $\Gamma$ is shown in the complex plane on Fig.1 for $\eta \rightarrow 0, R_{\Gamma} \rightarrow \infty$. One then rewrites :

$$
\begin{aligned}
\Re e\left(\mathcal{I}_{m n}+\mathcal{I}_{n m}\right) & =-\frac{i}{2 \pi} \oint_{\Gamma} d z\left(\int_{-\infty}^{\infty} d E \frac{\rho(E) \mathcal{P}_{n}(E)}{z-E}\right) \times \\
& \times\left(\int_{-\infty}^{\infty} d E^{\prime} \frac{\rho\left(E^{\prime}\right) \mathcal{P}_{m}\left(E^{\prime}\right)}{z-E^{\prime}}\right)
\end{aligned}
$$

By application of Jordan Lemme, the integral on the contour $\Gamma$ tends to zero when the radius goes to infinity, and it only remains four integrals on the real axis respectively for $[-\infty, a-2 b],\left[a-2 b, E_{F}\right],\left[E_{F}, a+2 b\right],[a+2 b,+\infty]$. Using the relation between first and second order Chebyshev polynomials defined on $[-1,+1]$ and associated with $\rho(E)=\sqrt{1-E^{2}}$ 


$$
\begin{gathered}
\lim _{\eta \rightarrow 0^{ \pm}} \int_{-1}^{+1} \frac{\sqrt{1-E^{2}} P_{n}(a+2 b E)}{\omega+i \eta-E} d E= \\
\pi\left\{Q_{n+1}(\omega) \mp i \pi \sqrt{1-\omega^{2}} P_{n}(\omega)\right\}
\end{gathered}
$$

it is easy to show that for $|\omega| \leq 1$, with $\omega=\cos \phi$ then

$$
\begin{aligned}
Q_{n}(\omega) & =\cos n \phi \\
P_{n}(\omega) & =\frac{\sin (n+1) \phi}{\sin \phi}
\end{aligned}
$$

and finally

$$
\lim _{\eta \rightarrow 0^{ \pm}} \int_{-1}^{+1} \frac{\sqrt{1-E^{2}} P_{n}(a+2 b E)}{\omega+i \eta-E} d E=\pi \exp (\mp i(n+1) \phi)
$$

In conclusion, given that the integration outside $[-1,1]$ leads to pure imaginary terms, the calculation of $\mathcal{I}_{m n}$ reduces to

$$
\begin{aligned}
\Re e\left(\mathcal{I}_{m n}+\mathcal{I}_{n m}\right) & =\frac{1}{2 \pi b} \int_{\frac{E_{F}-a}{2 b}}^{1} d \omega \sin ((m+n+2) \phi) \\
& -\frac{1}{2 \pi b} \int_{-1}^{\frac{E_{F}-a}{2 b}} d \omega \sin ((m+n+2) \phi) \\
& =\frac{1}{2 \pi b}\left(\frac{\sin (m+n+3) \mathcal{A}_{F}}{m+n+3}-\frac{\sin (m+n+1) \mathcal{A}_{F}}{m+n+1}\right)
\end{aligned}
$$

This can be done by noticing that n-recursion steps gives $\frac{n}{W}$ (W the total bandwith) as a resolution in energy., and that the states that will mainly contribute to RKKY, are enclosed in an interval of width $\Delta E \leq \hbar / \tau_{L}$ around Fermi energy. Here, we define $\tau_{L}$ as the time needed for electrons to travel between two magnetic impurities distant of L. For a sufficient resolution of our spectrum, the number of recursion steps to be considered is $n \gg$ $W \tau_{L} / \hbar$.

This result may also be recovered by looking at the propagation of recursion states in real space. In fact, the propagation of the $n$-th recursion state turns out to be representative of the real wave-function at a time $t \sim n \hbar / W,\left|\varphi_{i}^{n}\right\rangle \sim\left|\Psi\left(t_{n} \sim n \hbar / W\right)\right\rangle$, initially located at the same site $\left|r_{i}\right\rangle$. Then, one has to check that the diffusion length of $\left|\varphi_{i}^{n}\right\rangle$, which we define as $\xi(n)$, is sufficiently larger than the distance between magnetic sites. Therefore as soon as $\xi(n) \gg L$, the coefficients $\alpha_{i j}^{k>n}$ will not contribute significantly.

The criterion $n \gg W \tau_{L} / \hbar$ will depend on the nature of propagation through the scattering time. For ballistic regimes (crystals), $\tau_{L} \sim v_{F}^{-1} . L$ with $v_{F}$ the Fermi energy, and $n \gg\left(W / \hbar v_{F}\right) . L$, whereas diffusive regimes will be associated to $L^{2}=D \cdot \tau_{L}$, with $D$ the diffusion constant and $n \gg(W / \hbar D) L^{2}$. Anomalous diffusion described by $L \sim A \tau^{\beta}(A, 0 \leq \beta<0.5$, or $0.5<\beta<1$ depending on the model) will lead to $n \gg(W / \hbar A) \cdot L^{1 / \beta}$ to be control empirically.

To test the efficiency of the method, we have checked that the susceptibility for tight-binding electrons with Fermi energy close to the band edges was equivalent to that of free electrons, i.e. well described by the law $\chi(r) \sim\left(2 k_{F} r \sin \left(2 k_{F} r\right)-\cos \left(2 k_{F} r\right)\right) /\left(k_{F} r\right)^{3} \quad$ in 2dimension). Random systems have also been simulated, by considering site energies distributed at random within $[-W, W]$. For distance larger than the mean free path, the decrease of the averaged susceptibility was found to be proportional to $\frac{1}{r^{2}} \exp \left(-r / l_{p m}\right)$, and the numerical value of the mean free path was in good agreement with the expected one. Calculations were performed for several values of $W$ with average over 150 configurations.

\section{APPLICATIONS}

To achieve the convergence of the calculation, one must ensure first of all, that the total length of the system is much larger that the distance between magnetic sites if one wants to consider an infinite medium. To close the system, we take periodic boundary conditions. Second, the numerical accuracy must be checked. We found that single precision was sufficient and gave the same results as double precision.

It remains to figure out how many recursion steps are needed (summation on $\mathrm{n}$ and $\mathrm{m}$ in the above formula).

\section{A. 2-D Tight-Binding simulation of a Hume Rothery Band Alloy}

In the following, we exemplify the role of local environment on RKKY interaction in a $2 \mathrm{D}$ system. Starting from a perfect crystal, we consider how perturbation of local order in the vicinity of the magnetic site may affect RKKY coupling on larger (mesoscopic) scales. The local 
disorder will be designed by a proper modification of local tight-binding parameters of the corresponding hamiltonian. By doing so, we ensure that the LDoS around the initial state $\left|\psi_{0}\right\rangle$ is strengthened in regards to TDoS at the same energy.

$$
\begin{aligned}
\mathcal{H} & =\sum_{|i j\rangle}|i j\rangle \varepsilon_{i j}\left\langle i j\left|+\gamma \sum_{|i j\rangle}\right| i j\right\rangle(\langle i j+1|+\langle i j-1| \\
& +\left\langle i+1 j|+\langle i-1 j|)+\tau \sum_{|i j\rangle} \mid i j\right\rangle(\langle\{i \pm 1 j \pm 1\}|)
\end{aligned}
$$

Our model (Fig.2) features two different hopping integral between first $(\gamma)$ and second $(\tau)$ nearest neighbors as well as an alternate distribution of site energies

$$
\epsilon_{i j}=\frac{\epsilon_{A}}{2}\left(1+(-1)^{i+j}\right)+\frac{\epsilon_{B}}{2}\left(1-(-1)^{i+j}\right)
$$

in the $\tau=0$ case, this leads to a two-band DOS with a real gap whose bandwith $\Delta=\epsilon_{B}-\epsilon_{A}\left(\epsilon_{A}<\epsilon_{B}\right)$.

The orthonormal recursion basis $\left|\psi_{n}\right\rangle$ and coefficient can be evaluated iteratively. Nonetheless, even for simple tight-binding models, the complexity rapidly increases 12. Taking $\varepsilon_{A}=0.1, \varepsilon_{B}=0.3, \gamma=0.1, \tau=0.09$ has TB parameters one gets a TDoS and a RKKY interaction as shown in the inset of Fig.3, and one notices in particular that the RKKY coupling shows a structure that manifests the two underlying periods of the lattice.

\section{B. Effect of local environment on RKKY}

Let us now elaborate on the nature of the local perturbation around a given magnetic sites. The idea is to particularize the local density of states LDoS around one given site and to measure the effect on the susceptibility on mesoscopic scale.

Concretely, we modify the fourth first shells around the initial site $\left|\psi_{0}\right\rangle$ in the following way $\gamma^{\prime}=0.65 \gamma, \tau^{\prime}=$ $1.26 \tau$ and the site energies respectively $\varepsilon_{A, B}^{\prime}=\varepsilon_{A, B}+$ $\lambda_{i}, \quad i=\{1,2,3,4\}$ with $\lambda_{1}=0.15, \lambda_{2}=0.12, \lambda_{3}=$ $0.09, \lambda_{4}=0.06$. Initial site energy is $\varepsilon_{A}=\left\langle\psi_{0}|\mathcal{H}| \psi_{0}\right\rangle$. Thereby, the corresponding $\operatorname{LDoS}\left(\rho_{i}(E)=-2 / \pi \Im m\left\langle r_{i}\right|\right.$ $\left.G(z)\left|r_{i}\right\rangle\right)$ is increased if Fermi energy is $E_{F}=0.176 \mathrm{eV}$ $\left(\rho_{i}(E) \geq \rho_{\text {Bulk }}(E)\right)$ as shown on Fig.4. The number of sites considered for the computation of the susceptibility in the numerical was about 250.000, and the number of recursion steps around $\sim 200$.

The calculated electronic susceptibility for the homogeneous and inhomogeneous cases are plotted on Fig.5 from the results, one clearly sees that such enhancement of LDoS leads to an increase of the susceptibility. This effect may be at the origin of peculiar magnetic properties [11] in quasicrystals where atomic order is known to be complex on mesoscopic scales. Assuming that local environments of magnetic sites are associated with strong local densities of states (when compared to the average total density of states), then the RKKY coupling could be anomalously strong when compared with other metallic disordered phases 13]. One notes that such strong fluctuations of LDoS at a given energy from site to site are also thought to be important at the proximity of a metal-insulator transition [5].

\section{Anomalous diffusion and RKKY in quasiperiodic systems}

General properties of electronic susceptibility in quasiperiodic systems are difficult to describe when compared with periodic ones. Indeed, the simple law obtained for free itinerant electrons may be questionable due to intrinsic incommensurability. We show in this section, that the use of recursion method gives here some unique informations about RKKY in quasiperiodic structures, and further provide a framework for investigating mesoscopic interaction in aperiodic systems. Hereafter we consider a 2D-Fibonacci quasilattice for which we will analyse the susceptibility in relation with the spectral properties on larger systems, by varying the length of our systems (25.000 sites), number of recursion steps (up to $500)$ and the intensity of quasiperiodic potentials. Corresponding spectral structure may be found elsewhere [18]. The hamiltonian is defined for a Fibonacci-2D quasilattice and written in tight-binding basis as

$$
\mathcal{H}=\sum_{i} \varepsilon_{i}\left|\mathbf{r}_{i}\right\rangle\left\langle\mathbf{r}_{i}\left|+\gamma \sum_{\langle i j\rangle}\right| \mathbf{r}_{i}\right\rangle\left\langle\mathbf{r}_{j}\right|
$$

for which site energies are given by $\varepsilon_{i}=\varepsilon_{i x}+\varepsilon_{i y}$, with $\varepsilon_{i x}, \varepsilon_{i y}= \pm V_{q p}$ (potential strength) according to a Fibonacci sequence. Hopping integrals are set constant for simplicity ( $\langle i j\rangle$ denotes first neighbors). On Fig.6, the TDoS for a 2D quasiperiodic Fibonacci quasilattice, as well as typical signature of incommensurate long range order are reported. The strength of the quasiperiodic potential is $V_{q p}=0.7(\gamma)$ and the susceptibility is given as a funtion of the distance between interacting magneticsite (in a-units, with a the lattice spacing). From an analysis as a function of the potential strength, we found that no Fermi wavelength can be properly defined and oscillations exhibit resurgences that are absent from the periodic potential. This is a surprising pattern absent for periodic potentials for which unique wave-vector (at Fermi level) and continuous decreasing of the coupling is found. Such patterns may however remember the local fluctuations found that we found in random systems for a given configuration of disorder. This may thus appear as 
a common feature between the RKKY in quasiperiodic and disordered systems on mesoscopic scales.

Incommensurability effects on the period can be revealed by analysis of the fluctuations of $\log \left|\chi\left(r_{i}-r_{j}\right)\right|$ as a function of $\log \left(r_{i}-r_{j}\right)$. Indeed, if the $V_{q p}=0.0$ case manifests the oscillating behavior $\cos \left(2 k_{F}|r|\right)$ with unique wave-vector, quasiperiodic potential breaks this pattern even for small values such as $V_{q p}=0.05(\gamma)$. On Fig. 7, we compare three results obtain at $E_{F}=-1.9 \gamma$ and $V_{q p}=0.0,0.05,0.1$. Appearance of site-dependent incommensurate phase shift is thus illustrated and unveil the action of quasiperiodic potential on electronic coherence $(\log |\chi|$ has been rescaled for more clarity)

We now consider the long range properties of RKKY in these systems. To that end, let us return to the relation between electronic localization and diffusion modes at the origin of interchange coupling. In the classical case (Brownian motion), assuming $p(\mathbf{r}, t)$ the probability density of finding a random walker (or a classical electron) at $\mathbf{r}$ after time $t$, the anomalous diffusion regime (found in a disordered fractal) is expressed by $\left\langle\mathbf{r}^{2}(t)\right\rangle_{\text {dis }}=\int \mathbf{r}^{2} p(\mathbf{r}, t) d^{D} \mathbf{r} \sim t^{2 \nu}$ [15]. Anomalous regime can also be found in quantum diffusion, especially in quasiperiodic systems 9, 10] and at the metal-insulator transition (quantum Hall systems) 16. This has been connected with multifractal properties of eigenstates. Indeed if we consider the spreading of wave-packet constructed from multifractal eigenstates, one finds numerically that $\left\langle\hat{\mathbf{r}}^{2}\right\rangle=\left\langle\Psi(t)\left|\hat{\mathbf{r}}^{2}\right| \Psi(t)\right\rangle=\int \mathbf{r}^{2}|\Psi(\mathbf{r}, t)|^{2} d^{D} \mathbf{r} \sim t^{2 \nu}$ with $\nu$ an exponent characterizing the strength of the potential, the intrinsic correlations, etc. According to an argument by E. Akkermans [17] the power-law decreasing of the averaged second moment of electronic susceptibility should not be affected by anomalous diffusion and remains of type $\left\langle\chi\left(\left|\left(r_{i}-r_{j} \mid\right)\right\rangle \sim\left|r_{i}-r_{j}\right|^{-D}\right.\right.$ for D-dimensional disordered systems.

For the quasiperiodic strengthes considered in our calculation (from $V_{q p}=0.05(\gamma)$ up to $V_{q p}=1.1(\gamma)$ ), the long-range power law is not affected qualitatively and one recovers the $1 /|r|^{-D}$ with $D=2$. This is exemplified on Fig.8 for two different intensities $V_{q p}=0.1$ and $V_{q p}=1.1$ compared with the long ranged oscillations for no quasiperiodic potential which mimic the periodic potential. Note that the slight departure from power-law in the case $V_{q p}=1.1$ (and for large distance) is due to finite-size effects and can be smoothed out by increasing recursion steps.

One remarks that results on the octagonal quasiperiodic tiling have been obtained previously [19], and also seem to indicate a significant site dependence of the electronic susceptibility apparently without alteration of the general power-law as a function of distance between magnetic sites.
These results are in agreement with the argument we gave in section II for the general dependence of $\chi\left(r_{i}, r_{j}\right)=\left(2 \rho\left(E_{F}\right)\right) / L^{D} \Re e \sum_{n, m} \Phi(n) \Phi^{*}(m) /(n+m)$ which gives a correct interpretation of the universal shape of $L^{-D}$ for a metallic system. But our assumption made on the level distribution does not include any subtle correlations in the spectrum, as those found at the metalinsulator transition and associated to a specific level spacing distribution 20]. If quasiperiodic potentials induces multifractal states, their general level spacing distribution has been however found to be described by the Gaussian Orthogonal Ensemble (GOE) of the Random Matrix theory 21. This involves a metallic state of the conduction system and our calculations are consequently in agreement with other recent results obtained on quasiperiodic systems. Manifestations of multifractality of eigenstates may be rather revealed by strong fluctuations of local densities of states [5].

To finish with, one notes that unexpected feature has been also revealed from careful analysis of site dependent susceptibility (to be published elsewhere). For a potential $V_{q p}=0.1$ and Fermi energies $E_{F}=-0.65(\gamma)$ (resp. $E_{F}=-0.575(\gamma)$ ) situations where the electronic coupling is purely ferromagnetic (resp. antiferromagnetic) were found [12]. Such kind of pattern unveil unprecedented signature of complicated localization effects unique to quasiperiodic structures. Related phenomena such as Kondo effect in quasiperiodic systems has been discuss in regards to the same localization effects [22].

\section{CONCLUSION}

A method to investigate RKKY interaction in periodic or aperiodic systems has been presented. Using real-space schemes, this approach enables in particular to analyse the effect of local inhomogeneities, quasiperiodic potential and by extension in all situations where usual diagonalization methods may be limited. Some conclusive check of the method have been given in comparison to expected behavior and specific models have been studied in order to exemplify the interest of our method. In particular we have shown that even strong regimes of localization induced by a quasiperiodic potential do not lead to a qualitative departure from general power-law dependence, and LDoS fluctuations could affect the intensity of the interaction.

\section{ACKNOWLEDGMENTS}

S.R is indebted to Prof. T. Fujiwara from Department of Applied Physics of Tokyo University for his kind hospitality and continuous support. This work has been 
conducted within the GERMA project (Groupe d'Etudes et de Recherches sur les Materiaux Avances).

[1] R. Haydock, Solid state Physics vol 35, ed F. Seits, D. Turnbull and H. Ehrenreich (New York academic) 216 (1980). The recursion method and its applications Editors D.G. Petitfor and D.L. Weaire (Springer Verlag), Springer Series in Solid State Sciences 58 (1984).

[2] M.A. Ruderman and C. Kittel, Phys Rev. 96, 99 (1954). T. Kasuya, Phys. Rev. 106, 893 (1957). K. Yosida, Prog. Theor. Phys. 16, 45 (1956).

[3] P.G. De Gennes, J. Phys. Radium 23, 630 (1962). G. Bergmann, Phys. Rev. B 36, 2469 (1987). L.N. Bulaevskii and S.V. Panyukov, JETP Letter 43, 240 (1986).

[4] A. Jagannathan, E. Abrahams and M.J Stephen, Phys. Rev. B, 37 (1988) 436.

[5] I.V. Lerner, Europhys. Lett., 16 (5), 479-484 (1991).

[6] F. Matsubara and M. Iguchi, Phys. Rev. Lett. 68, 3781 (1992)

[7] J.J. Rodriguez-Nuñez, H. Beck, J. Konior, A. M. Oles and B. Coqblin, Phys. Lett. A 197, 173 (1995).

[8] P. Bruno and C. Chappert, Phys. Rev. Lett. 67, 1602 (1991). B.A. Jones and C.B. Hanna, Phys. Rev. Lett. 71, 4253 (1993). P. Bruno, J. Kudrnovsky, V. Drchal and I. Turek, Phys. Rev. Lett. 76, 4254 (1996).

[9] S. Roche and D. Mayou, Phys. Rev. Lett. 79, 2518 (1997).

[10] S. Roche, G. Trambly de Laissardiere and D. Mayou, J. Math. Phys. 38, 1794 (1997).

[11] C. Berger and J.J. Préjean, Phys. Rev. Lett. 64, 1769 (1990); A. Gozlan et al, Phys. Rev. B 44, 2 (1991).

[12] S. Roche, Ph.D. Thesis, University Joseph-Fourier (1996), unpublished.

[13] S. Roche and D. Mayou, Proc. of the 5th International Conference on Quasicrystals, Avignon, 1995, Editors C. Janot, R. Mosseri, 413 (World Scientific, 1995).

[14] G. Szegö: Orthogonal polynomials 4th ed. Colloquium Publications 23 (Providence, Rhode Island: Am. Math. Soc. 1975).

[15] S. Havlin and D. Ben Avraham, Adv. Phys. 36, 695 (1987). J.P. Bouchaud and A. Georges, Physics Reports 195, 131 (1990)

[16] B. Huckestein and L. Schweitzer, Phys. A 191, 406 (1991). Takamichi Terao, Tsuneyoshi Nakayama and H. Aoki, Phys. Rev. B 54, 10350 (1996).

[17] E. Akkermans (unpublished work).

[18] X. Fu, Y. Liu, B. Cheng and D. Zheng, Phys. Rev. B 43, 10808 (1991).

[19] A. Jagannathan, J. Phys. I (France) 4, 133 (1994).

[20] I.K. Zharekeshev and B. Kramer, Phys. Rev. Lett. 79, 717 (1997).

[21] J.X. Zhong, U. Grimm. R.A. Romer and M. Schreiber, Phys. Rev. Lett. 80, 3996 (1998).

[22] V.G. Benza and E. Montaldi ,J. Phys. A: Math. Gen. 27, 2299-2304 (1994).

\section{FIGURES CAPTIONS}

Fig. 1. Integral contour for the calculation of the coefficient $\mathcal{I}_{m n}$ and semi-elliptic density of states $\rho_{a b}(\omega)$ used in the case of Chebyshev polynomials.

Fig. 2. Schematic representation of the lattice with different tight-binding parameters.

Fig. 3. Total density of states for the hamiltonian in the perfect Hume-Rothery alloy and corresponding RKKY coupling (inset).

Fig. 4. Local density of states around the modified local environment of one magnetic site.

Fig. 5. Comparison of the electronic susceptibility (in a-units, a lattice parameter) for the homogeneous (bold line) and the inhomogeneous cases.

Fig. 6. RKKY coupling for a quasiperiodic system $\left(V_{q p}=0.7\right)$ and different Fermi energies. The inset shows the corresponding total density of states.

Fig. 7. Electronic susceptibility as a function of $\log |\chi|$ for different small quasiperiodic potentials $\left(V_{q p}=\right.$ $0,0.050 .01$ )

Fig. 8. (a) Electronic susceptibility as a function of $\log |\chi|$ for small and strong quasiperiodic potentials $\left(V_{q p}=0.11 .1\right)$ compared to periodic potential. The inset (b) shows the local susceptibility for $V_{q p}=0.1$, the same Fermi energy and for several different environment between magnetic sites in the quasiperiodic potential. 


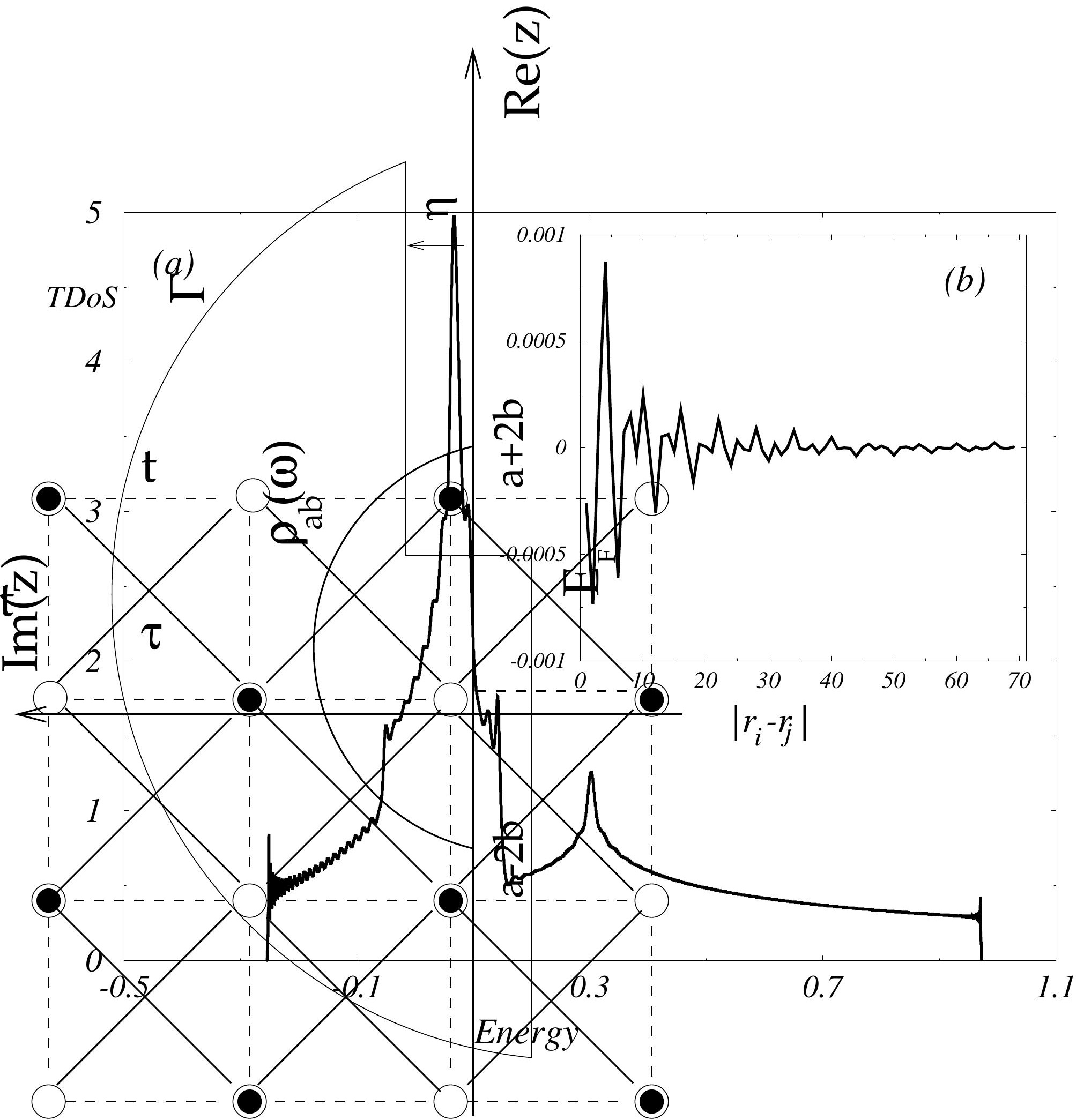




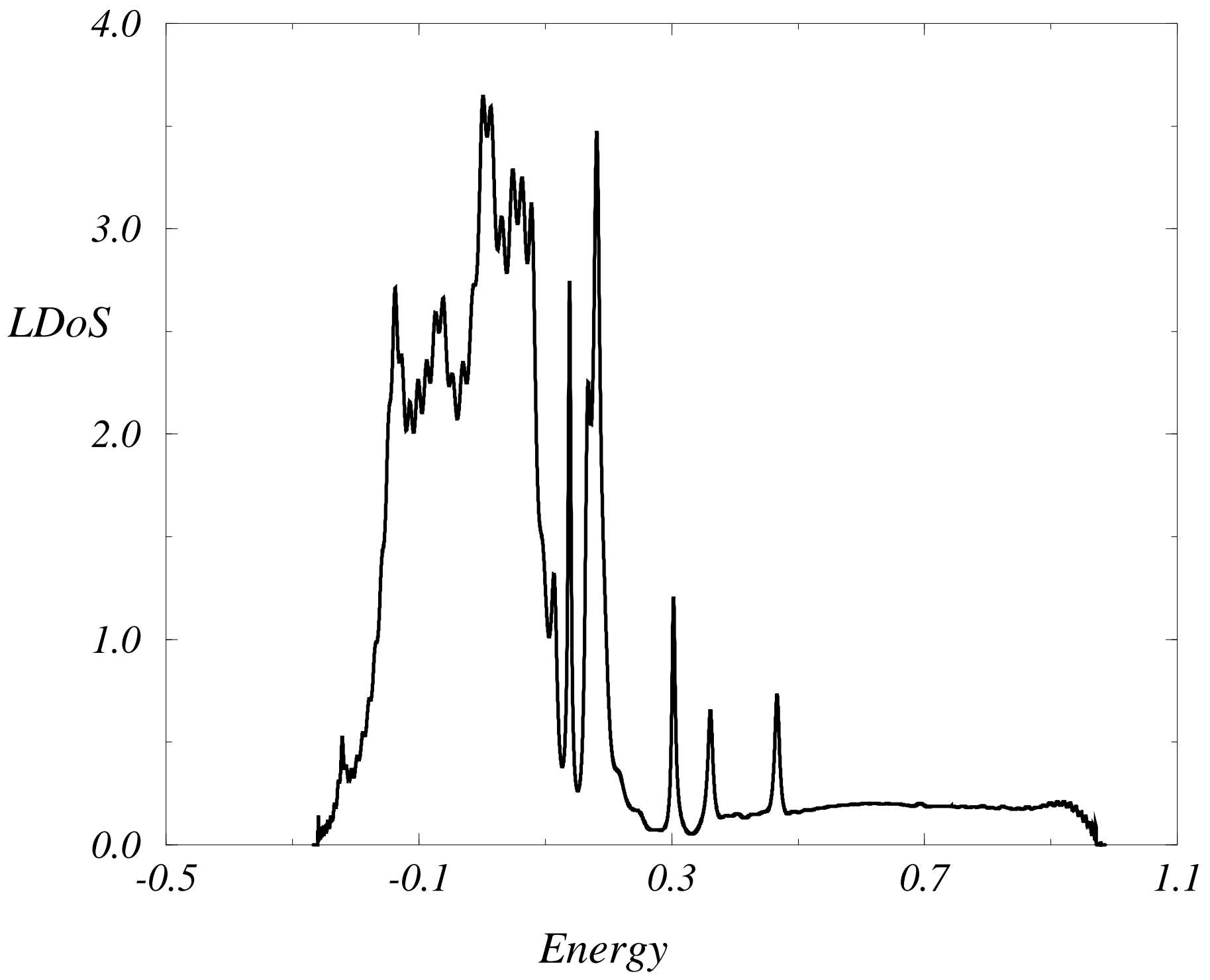




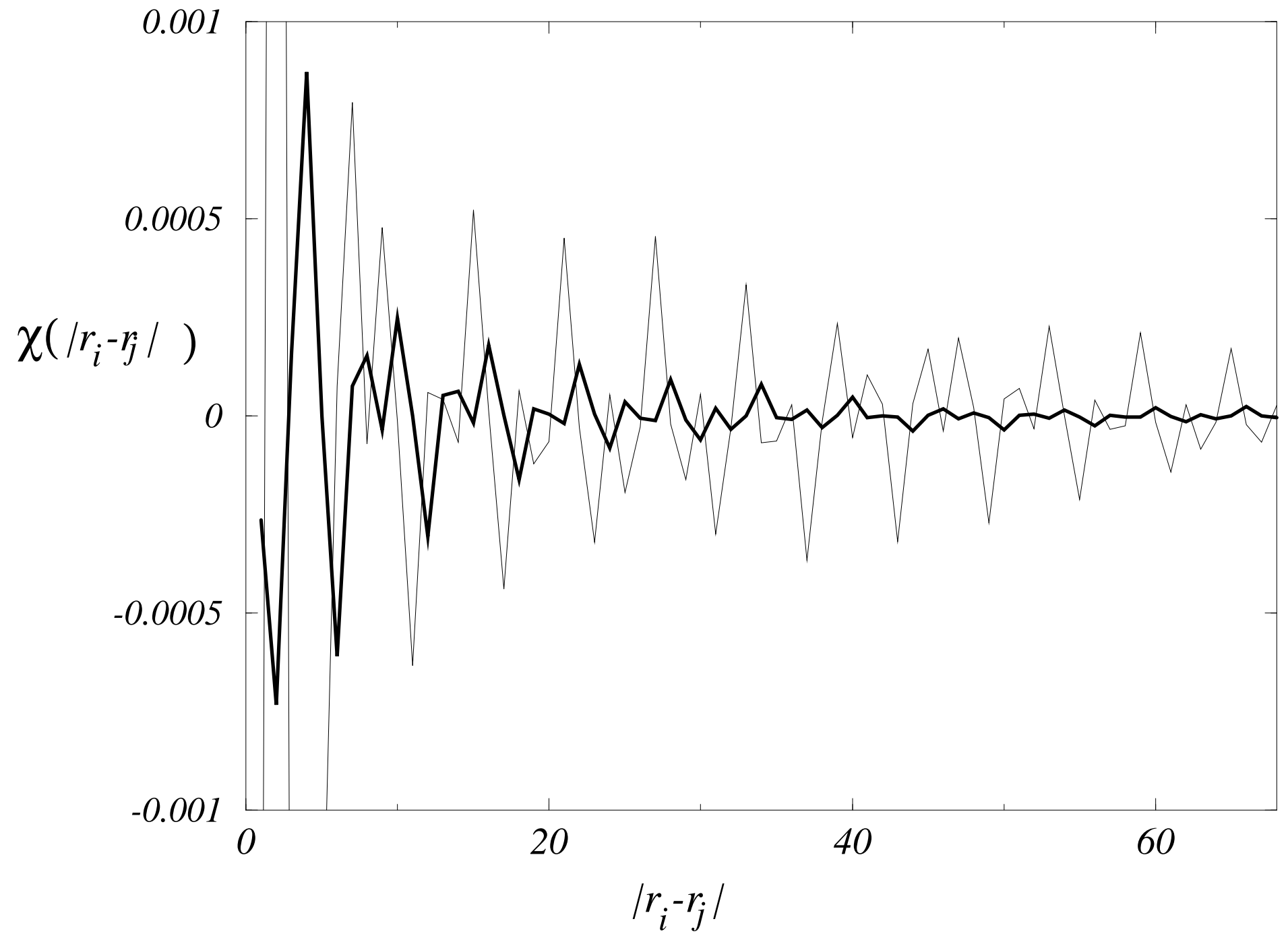




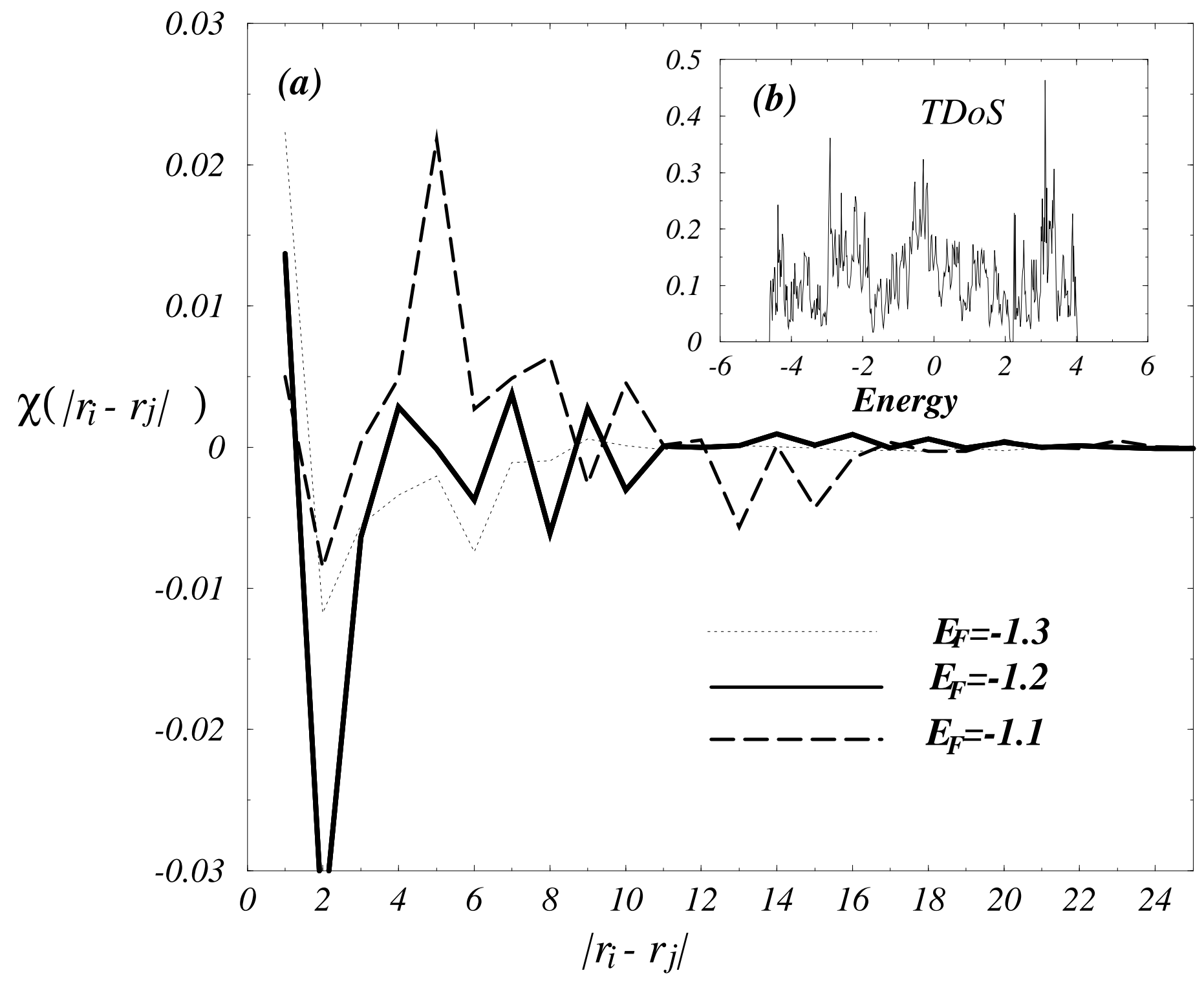




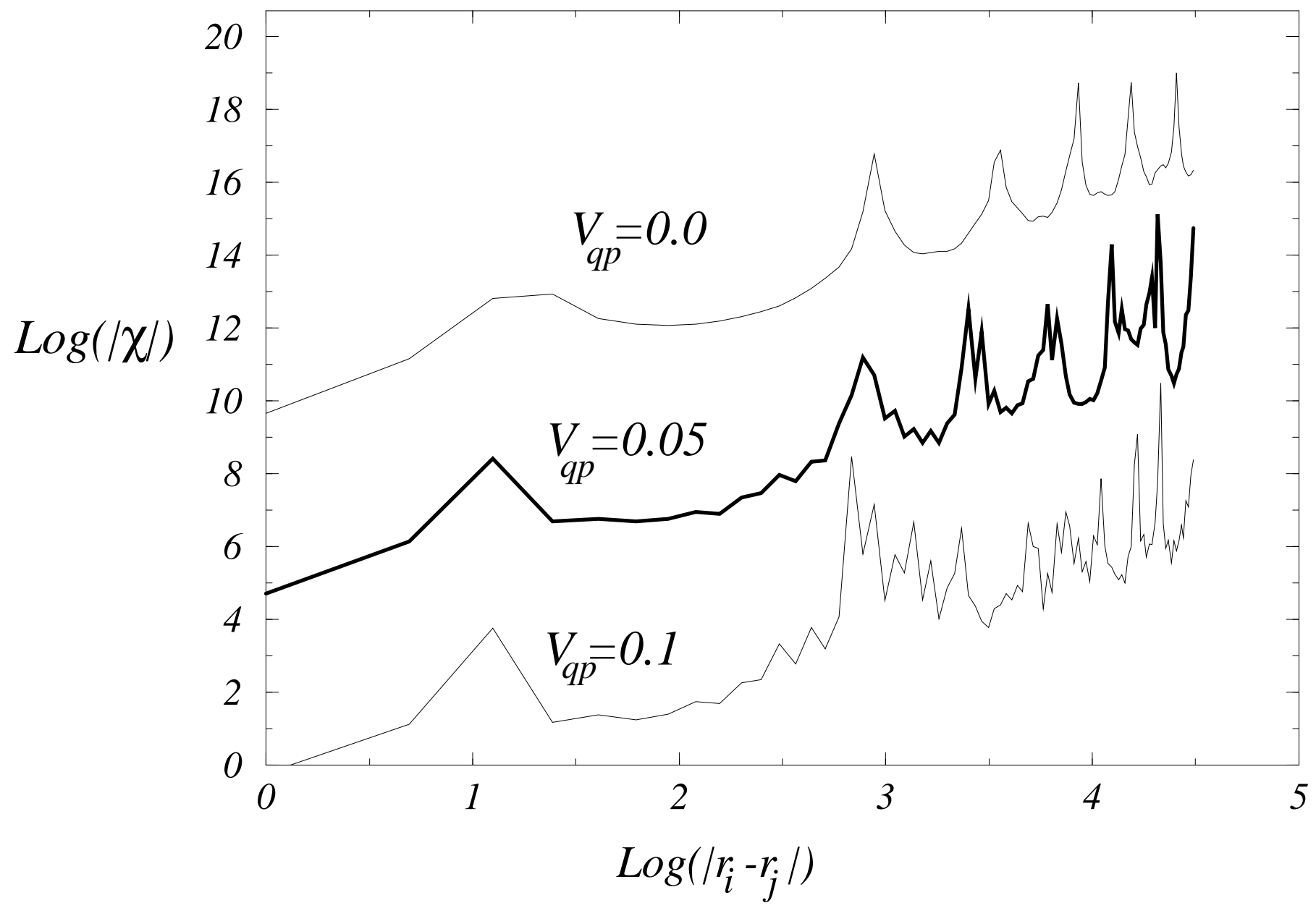




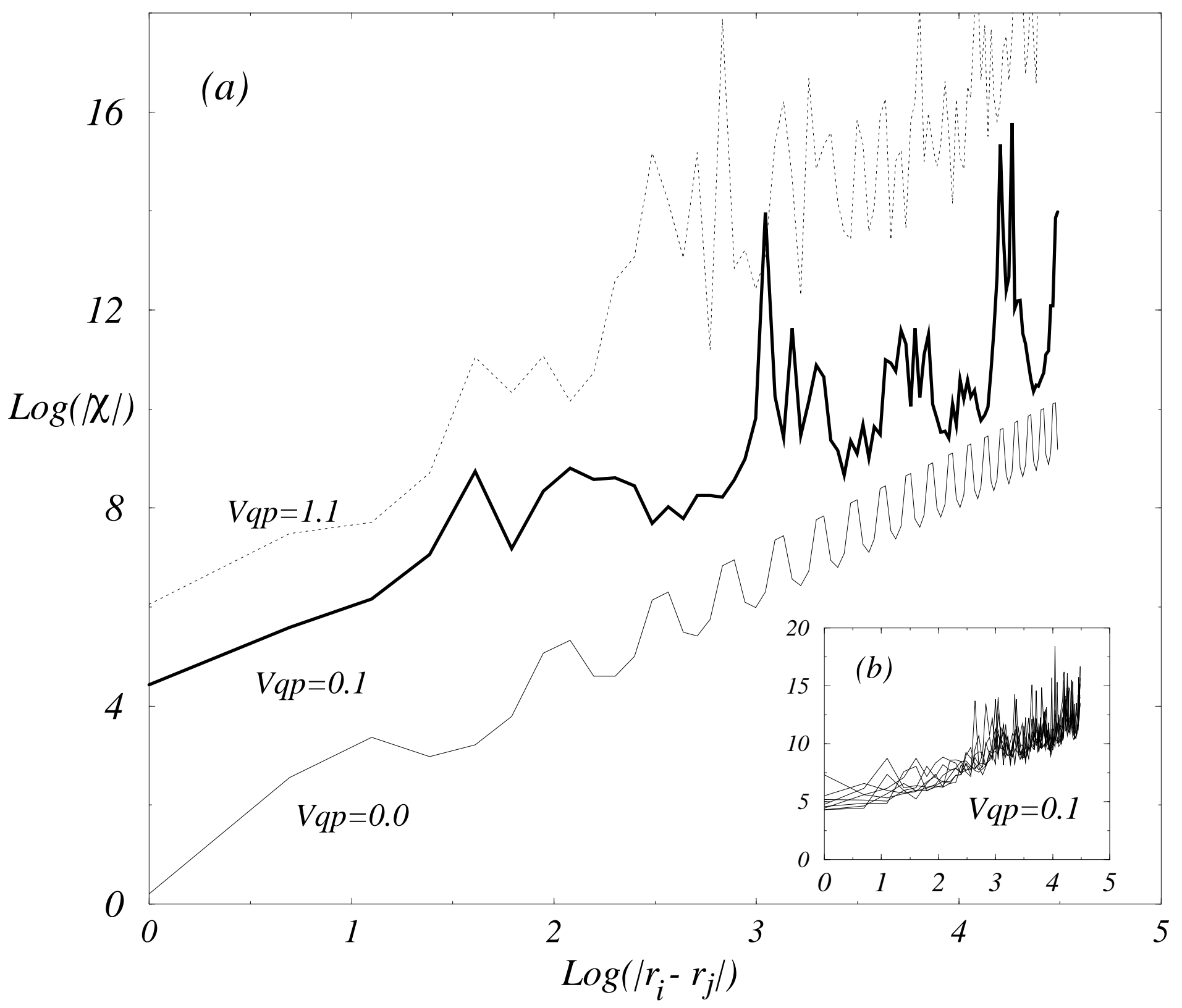

